\title{
HEART RATE REDUCTION WITH ESMOLOL IS ASSOCIATED WITH IMPROVED ARTERIAL ELASTANCE IN PATIENTS WITH SEPTIC SHOCK. A PROSPECTIVE OBSERVATIONAL STUDY
}

\section{Esmolol reduces arterial elastance in septic shock}

A Morelli MD ${ }^{1}$, M Singer MD, FRCP ${ }^{2}$, VM Ranieri MD ${ }^{1}$, A D’Egidio MD ${ }^{1}$, L Mascia MD ${ }^{4}, A$ Orecchioni MD ${ }^{1}$, F Piscioneri ${ }^{1}$, F Guarracino MD ${ }^{3}$, E Greco MD ${ }^{1}$, M Peruzzi MD ${ }^{4}$, G BiondiZoccai MD ${ }^{4.5}$, G Frati MD $^{4,5}$, SM Romano MD ${ }^{6}$

${ }^{1}$ Department of Cardiovascular, Respiratory, Nephrological, Anesthesiological and Geriatric Sciences, University of Rome, “La Sapienza”, Policlinico Umberto Primo, Viale del Policlinico 155, Rome 00161, Italy; ${ }^{2}$ Bloomsbury Institute of Intensive Care Medicine, University College London, Cruciform Building, WC1E 6BT London, United Kingdom; ${ }^{3}$ Cardiothoracic Anesthesia and Intensive Care Medicine, Dept of Anesthesia and Intensive Care, University Hospital of Pisa, via Roma 55 - 56126 Pisa, Italy; ${ }^{4}$ Department of Medico-Surgical Sciences and Biotechnologies, University of Rome "La Sapienza”, Corso della Repubblica, 79 - 04100 - Latina, Italy; 5 Department of AngioCardioNeurology, IRCCS Neuromed, Via Atinense 18 - 86077 - Pozzilli, Italy

${ }^{6}$ Department of Experimental and Clinical Medicine, Unit of Internal Medicine and Cardiology, University of Florence, Largo Brambilla, 350134 Florence, Italy

\section{Corresponding Author}

Andrea Morelli, Department of Cardiovascular, Respiratory, Nephrological, Anesthesiological and Geriatric Sciences, University of Rome, "La Sapienza", Italy, Viale del Policlinico 155, Rome 00161, Italy E-mail: andrea.morelli@uniroma1.it Telephone: +39 0649978024, Fax: +39 0649978019 


\begin{abstract}
Purpose: Ventricular-arterial (V-A) decoupling decreases myocardial efficiency and is exacerbated by tachycardia that increases static arterial elastance (Ea). We thus investigated the effects of heart rate $(\mathrm{HR})$ reduction on Ea in septic shock patients using the beta-blocker esmolol. We hypothesized that esmolol improves Ea by positively affecting the tone of arterial vessels and their responsiveness to HR-related changes in stroke volume (SV).
\end{abstract}

Methods: After at least 24 hours of hemodynamic optimization, 45 septic shock patients, with a HR $\geq 95 \mathrm{bpm}$ and requiring norepinephrine to maintain mean arterial pressure (MAP) $\geq 65 \mathrm{mmHg}$, received a titrated esmolol infusion to maintain HR between 80-94 bpm. Ea was calculated as MAP/SV. All measurements, including data from right heart catheterization, echocardiography, arterial waveform analysis and norepinephrine requirements, were obtained at baseline and at 4 hours after commencing esmolol.

Results: The HR target was achieved in all patients and this was associated with a decrease in Ea $\left(2.19 \pm 0.77\right.$ vs. $\left.1.72 \pm 0.52 \mathrm{mmHg} \cdot 1^{-1}\right)$, arterial $\mathrm{dP} / \mathrm{dt}_{\max }\left(1.08 \pm 0.32\right.$ vs. $\left.0.89 \pm 0.29 \mathrm{mmHg} \cdot \mathrm{ms}^{-1}\right)$ and a parallel increase in stroke volume ( $48 \pm 14$ vs. $59 \pm 18 \mathrm{ml})$, all $\mathrm{p}<0.05$. Cardiac output and ejection fraction remained unchanged whereas norepinephrine requirements were reduced $(0.7 \pm 0.7$ to $\left.0.58 \pm 0.5 \mu \mathrm{g} \cdot \mathrm{kg}^{-1} \cdot \mathrm{min}^{-1}, \mathrm{p}<0.05\right)$.

Conclusions: HR reduction with esmolol effectively improved Ea while allowing adequate systemic perfusion in patients with severe septic shock who remained tachycardic despite standard volume resuscitation. As Ea is a major determinant of V-A coupling, its reduction may contribute to improving cardiovascular efficiency in septic shock.

Key words: tachycardia; septic shock; receptors, adrenergic, beta; heart rate, arterial elastance 


\section{INTRODUCTION}

Despite achieving recommended hemodynamic targets [1], ventricular-arterial (V-A) decoupling may persist in patients with septic shock [2,3]. This decoupling is further exacerbated both by an increase in afterload through administration of vasoconstrictor agents, [2-4] and by tachycardia [5,6]. Decoupling is associated with cardiovascular inefficiency and unfavorable myocardial energetics that can deteriorate progressively during the course of the disease [2-6]. Tachycardia also increases myocardial oxygen consumption and, by shortening diastolic relaxation time, may impair coronary perfusion; these effects may further contribute to myocardial dysfunction and a poor outcome [7-12].

We recently reported the impact of the short-acting selective beta ${ }_{1}$-adrenergic blocker esmolol on hemodynamics in a cohort of critically ill, fluid-resuscitated, septic shock patients with persistent tachycardia and high catecholamine requirements [10]. The esmolol-induced decrease in heart rate (HR) was associated with improved hemodynamics and reductions in norepinephrine dosing and troponin levels. This improvement may be related, at least in part, to enhanced diastolic function and/or better V-A coupling $[13,14]$.

As arterial tone is a major determinant of $\mathrm{V}$-A coupling $[2,3,13,14]$, HR reduction may improve V-A coupling by decreasing static arterial elastance (Ea) $[13,14]$. As Ea is linearly determined by stroke volume (SV), any improvement in diastolic time and filling can directly influence Ea. The purpose of the present prospective observational study was to investigate the effects of HR reduction on Ea in tachycardic septic shock patients. We hypothesized that esmolol improves Ea by positively affecting the tone of arterial vessels and their responsiveness to HR-related changes in stroke volume. 


\section{METHODS}

\section{Patients}

The study was undertaken in an 18-bed multidisciplinary intensive care unit (ICU) ("La Sapienza", University of Rome, Policlinico Umberto I). An institutional review board provided ethical approval for the study (ClinicalTrials.gov NCT02188888). Enrolment occurred between December 2013 and June 2014. Consent was waived as patients at study entry were not able to provide informed consent. Permission to use collected data was obtained from the patient when mental competency was regained or, otherwise, their next-ofkin.

We enrolled septic shock patients who, despite adequate fluid resuscitation, were still requiring high-dose norepinephrine (NE) to maintain a mean arterial pressure (MAP) $\geq 65$ mmHg [1], and had a tachycardia $>95$ beats per minute (bpm) after 24 hours [10].

Exclusion criteria were: age $<18$ years, cardiac dysrhythmias, need for an inotropic agent, significant valvular heart disease and pregnancy. All patients were sedated with remifentanil and propofol, and received volume-controlled mechanical ventilation.

\section{Hemodynamic measurements}

Systemic hemodynamic monitoring included pulmonary artery (7.5F, Edwards Lifesciences, Irvine, CA, USA) and radial artery catheterization. MAP, central venous pressure (CVP), mean pulmonary arterial pressure (MPAP), and pulmonary arterial occlusion pressure (PAOP) measured at end-expiration. HR was measured from continuous electrocardiographic recording. Cardiac output $\left(\mathrm{CO}^{\text {th }}\right)$ and stroke volume $\left(\mathrm{SV}^{\text {th }}\right)$ was estimated by the continuous thermodilution technique (Vigilance II ${ }^{\circledR}$, Edwards Lifesciences, Irvine, CA, USA). 
The radial artery catheter was connected to the MostCare ${ }^{\circledR}$ hemodynamic monitor (Vytech, Padua, Italy). This device utilizes the pressure recording analytic method (PRAM) to provide continuous beat-to-beat monitoring of stroke volume $\left(\mathrm{SV}^{\mathrm{p}}\right)$ with assessment of left ventricular function $[15,16]$. Measures obtained from analysis of the radial arterial pressure waveform contour were: systolic pressure $\left(\mathrm{P}_{\text {sys }}\right)$, diastolic pressure $\left(\mathrm{P}_{\mathrm{dia}}\right)$, dicrotic notch $\left(\mathrm{P}_{\mathrm{dic}}\right)$ and MAP, peripheral artery $\mathrm{dP} / \mathrm{dt}_{\max }\left(\operatorname{art} . \mathrm{dP} / \mathrm{dt}_{\max }\right)$, cardiac cycle efficiency $(\mathrm{CCE})[15,16]$. After data acquisition, we calculated differences between MAP and $\mathrm{P}_{\text {dic }}\left(\mathrm{MAP}-\mathrm{P}_{\mathrm{dic}}\right)[17,18]$ and cardiac power output (CPwO) [19].

For calculation of Ea we applied the formula MAP/SV [2-6,20]. SV was calculated by using the thermodilution technique in which $\mathrm{SV}^{\text {th }}=\mathrm{CO}^{\text {th }} / \mathrm{HR}$. This was directly estimated from a single beat obtained by arterial waveform analysis $\left(\mathrm{SV}^{\mathrm{p}}\right)[15,16]$.

\section{Echocardiography}

Two-dimensional real-time echocardiographic studies were performed to assess left ventricular ejection fraction (LVEF), using a wide-angle phased-array digital sector scanner and a 5-MHz multiplane transesophageal probe (T6H, HD7 XE Philips, Eindhoven, The Netherlands).

\section{Study design}

After at least 24 hours of hemodynamic stabilization targeted at achieving values of PAOP $\geq 12 \mathrm{mmHg}, \mathrm{CVP} \geq 8 \mathrm{mmHg}$, and MAP $\geq 65 \mathrm{mmHg}$ [1], those patients who remained with HR values $\geq 95 \mathrm{bpm}$ were treated with a continuous esmolol infusion to maintain HR between 80 $94 \mathrm{bpm}$ [10]. The protocol required a titrated esmolol infusion commenced at $25 \mathrm{mg} \bullet \mathrm{h}^{-1}$, with an upper dose limit of $2000 \mathrm{mg} \bullet \mathrm{h}^{-}{ }^{1}$, to maintain this predefined HR range [10]. During this intervention period, conventional treatment was continued as per usual practice. Fluid challenges were performed, and repeated as necessary, to maintain $\mathrm{CVP} \geq 8 \mathrm{mmHg}$ and $\mathrm{PAOP}$ 
$\geq 12 \mathrm{mmHg}$ [1]. Norepinephrine (NE) was titrated to maintain MAP $\geq 65 \mathrm{mmHg}$. None of the patients received inotropic support (levosimendan or dobutamine) during the intervention period. Hemodynamic and echocardiographic variables as well as NE requirements were determined at baseline and after four hours of esmolol infusion. Since our ICU guideline requires administration of esmolol to maintain the predefined HR threshold for the whole length of the patient's stay in ICU, death from any cause was assessed at Day 28 after esmolol commencement.

\section{Statistical analysis}

All hemodynamic variables were evaluated before and after esmolol administration using Student's t-test for paired data. Data were analyzed with IBM SPSS 20.0 statistical software (SPSS, Chicago, IL, USA). Values were reported as mean \pm standard deviation (SD) or absolute values. Statistical significance was established at a two-tailed P level $<0.05$.

\section{Role of the funding source}

This study was funded by an independent research grant from the Department of Anesthesiology and Intensive Care of the University of Rome "La Sapienza".

\section{RESULTS}

One hundred and sixteen septic shock patients were screened for enrollment and forty-five patients were included in the study (Fig 1). Their demographics and outcomes are summarized in Table 1. Twenty-two patients (49\%) survived until Day 28. Of note, their norepinephrine requirements were high as in our previous study (10) and all patients received esmolol to maintain the predefined HR threshold for the whole length of the patient's stay in ICU 
Hemodynamic and echocardiographic variables and norepinephrine requirements.

The target range of 80-94 bpm was not achieved in all patients after 4 hours of esmolol infusion, as HR values were collected while performing transesophageal echocardiography and this procedure did, in some patients, induce brief HR fluctuations. In six patients HR transiently rose (to $(101 \pm 1.7 \mathrm{bpm})$, while in 5 patients HR transiently fell (to $70 \pm 1.3 \mathrm{bpm}$ ) (Figure 2). The protocol did not allow any modification in the level of sedation (thereby influencing afterload). Compared to baseline, there were significant decreases (all $\mathrm{p}<0.001$ ) in $\mathrm{Ea}^{\text {th }}, \mathrm{Ea}^{\mathrm{p}} \mathrm{MAP}, \mathrm{MPAP}$ and norepinephrine requirements at the 4 hour timepoint, while $\mathrm{SV}^{\mathrm{p}}$ and $\mathrm{SV}^{\text {th }}, \mathrm{CCE}$, and $\mathrm{CPwO}$ (all $\mathrm{p}<0.01$ ). $\mathrm{CO}^{\mathrm{p}}$ and $\mathrm{CO}^{\text {th }}$ and $\mathrm{LVEF}$ remained unchanged. (Table 2). No differences were found when comparing $\mathrm{CO}^{\mathrm{p}}$ and $\mathrm{CO}^{\text {th }}$ as well as $\mathrm{SV}^{\mathrm{p}}$ and $\mathrm{SV}^{\text {th }}$ at the study timepoints.

Compared to baseline, while $\mathrm{P}_{\text {dic }}$ remained unchanged, $\mathrm{P}_{\text {sys }}, \mathrm{P}_{\text {dia }}$, MAP $-\mathrm{P}_{\text {dic }}$, and $\operatorname{art.dP} / \mathrm{dt}_{\max }$ significantly decreased after 4 hours' esmolol infusion (all $\mathrm{p}<0.001)$ (Table 2).

\section{DISCUSSION}

We demonstrate that an esmolol infusion, given over 4 hours and titrated to reduce HR below $95 \mathrm{bpm}$ in a cohort of fluid-resuscitated septic shock patients, was associated with a decrease in $\mathrm{Ea}$ and a parallel increase in SV. CO and LVEF remained unchanged whereas norepinephrine requirements were reduced.

The performance of the cardiovascular system depends on its ability to optimize coupling between the ventricle and arterial system $[2,3,20]$. In the presence of optimal V-A coupling, the cardiovascular system reaches its maximum efficiency; this implies that all the pulsating 
energy produced by the left ventricle is transmitted downstream to the peripheries with lower energy costs $[2,3,20]$.

Sympathetic activation plays a pivotal role in matching the ventricle to the arterial system. The consequent rise in HR leads to an increase in myocardial contractility to match the concomitant increase in afterload due to the force-frequency response $[2,3,20]$. By contrast, in the septic heart, alterations in sarcoplasmic reticulum calcium handling and $\beta$-adrenergic receptor downregulation abolish the force-frequency effect on myocardial contractility $[2,3,4$, 20,22]. Under such conditions tachycardia further decreases myocardial performance and thus worsens V-A coupling. Due to unfavorable cardiac energetics, such uncoupling will contribute to myocardial dysfunction [2-6].

An elevated HR is associated with an increased mortality risk in patients with septic shock [812]. In agreement with other clinical and experimental studies $[23,24]$, the reduction in HR with esmolol was accompanied by an increase in SV and did not cause significant cardiovascular derangement to any of our patients. For a similar preload, CO was not significantly reduced due to the concomitant increase in SV. Although MAP decreased, it remained within acceptable limits $[1,25]$ while a reduction was achieved in norepinephrine requirements.

The above findings confirm that, in the presence of an adequate preload, lowering HR allows better ventricular filling during diastole, hence increasing SV [7,23,24]. We hypothesized that beside the effects of HR reduction on diastolic function, the increase in SV was also the result of improved arterial loading as a consequence of a reduced Ea. In line with previous studies $[13,14]$, we noticed a decrease in Ea after esmolol administration. This presents the result of the interplay between wall stiffness, compliance and outflow resistance, and thus expresses the true afterload imposed on the left ventricle [2-6,20]. Of note, changes in SV occurred while reducing NE requirements but without any change in LVEF. This indicates that the 
increase in SV was not the result of increased myocardial contractility but of better ventricular filling and reduced Ea. As LVEF may be largely affected by afterload in septic shock, a decrease in Ea also explains the finding of an unchanged LVEF after esmolol infusion [31]. Changes in Ea may be mediated by both enhanced endothelial function and modifications of the physical properties of the vasculature. Beta-blockers may improve endothelium-dependent relaxation by exerting anti-inflammatory activity, reducing vascular oxidative stress and modulating nitric oxide pathways [23,24,26-28]. On the other hand, by acting on the viscoelastic component of the vessel wall, HR per se may affect the tendency of the arterial vessels to distend in response to their intravascular pressure [29,30]. Since vessel distensibility is time-dependent, arterial vessels will have more a rigid structure if the time allowed for them to distend is reduced, as occurs during tachycardia [29,30]. HR reduction can therefore lead to arterial de-stiffening and increased compliance [13,14].

Art.dP/dt $\mathrm{dax}_{\max }$ decreased on reducing $\mathrm{HR}$, while $\mathrm{SV}$ improved and NE requirements were reduced. These findings indicate that in addition to improved diastolic phase, reduced Ea allowed LV to generate a higher SV with less contractility and lower energetic cost (Fig 3). However, this decrease may be also caused by a direct effect of esmolol on myocardial contractility. To further elucidate the effects of HR reduction on cardiovascular performance we analyzed changes in MAP - $\mathrm{P}_{\text {dic. }}$ This is a true measure obtained from the pulse contour and accurately expresses the interaction between LV contractility and a given afterload $[17,18]$. This measure may indicate changes in contractility better than LVEF as the latter may be normal in septic shock despite a serious impairment of intrinsic LV contractility [31]. In healthy subjects the value of MAP - $\mathrm{P}_{\text {dic }}$ is very low as the level of MAP and $\mathrm{P}_{\text {dic }}$ are similar. By contrast, in septic shock the value of MAP - $\mathrm{P}_{\text {dic }}$ is high due to reduced arterial tone, reflecting an adaptive response to a decrease in SV. Indeed, a decrease in arteriolar resistance allows a higher SV at any given contractility and left ventricular filling. After HR 
reduction, we noticed a decrease in MAP - $\mathrm{P}_{\text {dic; }}$ this suggests that, in some patients, the afterload became potentially excessive for that level of contractility $[4,17,18]$. Nevertheless, even in this situation, HR reduction did not cause further cardiovascular derangement and the administration of esmolol was well tolerated. We hypothesized that an improved diastolic phase with better ventricular filling compensated for decreased contractility. It is therefore conceivable that, in some patients, improved diastolic function contributed more than the reduction in Ea towards increasing myocardial efficiency. Taken together, these results suggest that reducing HR in tachycardic septic shock patients with a beta-blocker will have variable effects, in particular on V-A coupling. This reinforces the need to individualize patient treatment according to both myocardial performance as well as their hemodynamic status.

As previously discussed, the increase in SV after $\mathrm{HR}$ reduction contributed to the maintenance of cardiac output. This altered hemodynamic profile should be considered as an economization of myocardial workload and oxygen consumption, thereby lowering the risk of myocardial ischemia. Accordingly, both CCE, a surrogate of cardiac function and cardiac power output (the strongest hemodynamic correlates of mortality in heart failure [19]), improved on reducing HR. An improvement in V-A coupling due to reduced Ea, together with an economization of myocardial workload and oxygen consumption may contribute to preserving myocardial efficiency, especially in established septic shock. Although this hemodynamic profile has been associated with improved outcomes (10), this hypothesis remains to be tested in larger trials.

There are several limitations to this study, the most important being that it was not designed as a randomized controlled trial. Such a design would best discriminate the effects of esmolol. Nevertheless, arterial elastance and myocardial performance can differ significantly between septic shock patients according to their preload, myocardial contractility, arterial stiffness and vasopressor support. Such variables and their relationships may vary considerably over time 
depending on disease progression. We therefore utilized a short-period observational design in which the patient served as his/her own control, rather than a randomized controlled design. We thus lack a control group so we cannot be certain that our findings are the consequence of the esmolol-induced reduction of HR rather than an independent evolution of the patient's condition. However, the brief observational period of 4 hours allowed us to limit this bias, and there was a marked consistency of response to esmolol. We did not investigate ventricular elastance, the other determinant of V-A coupling. An accurate measure of ventricular elastance is, however, extremely difficult to perform in severely compromised septic shock patients due to the invasiveness and the risks of the technique. For the same safety concern,

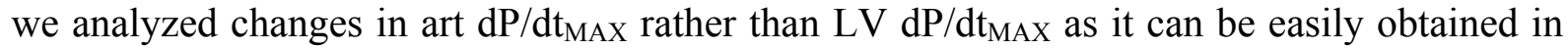
the majority of septic shock patients from their arterial pressure waveform. However, art $\mathrm{dP} / \mathrm{dtMAX}$ is a surrogate measure; in the presence of vasoplegia (as in septic shock) this may not accurately reflect $\mathrm{LV} \mathrm{dP} / \mathrm{dtmax}$.

Since we enrolled only patients in a hyperdynamic state and with preserved LVEF after at least 24 hours of hemodynamic optimization, we cannot extrapolate our results to earlier phases of septic shock or to patients with lower LVEF or CO, for whom further investigations are needed. We also did not measure longer term changes to assess the evolution in ventricular performance characteristics, although a potential bias due to other confounding influences will be greater.

\section{CONCLUSION}

Despite achieving recommended hemodynamic targets, V-A decoupling may persist in patients with septic shock and it can deteriorate progressively during the course of the disease [2-4]. Such patients may potentially benefit from therapies aimed at normalizing V-A coupling. Among them, HR reduction with esmolol could effectively improve Ea while 
allowing adequate systemic perfusion in septic shock patients remaining tachycardic despite standard resuscitation. As Ea is a major determinant of V-A coupling, its reduction may contribute to improving cardiovascular efficiency in septic shock.

Conflict of Interest Disclosures: Andrea Morelli received honoraria for speaking at Baxter symposia. Mervyn Singer served as a consultant and received honoraria for speaking and chairing symposia for Baxter. Salvatore Mario Romano has a patent METHOD AND APPARATUS FOR MEASURING CARDIAC FLOW OUTPUT (USA Patent Number 6758822). No other disclosures were reported.

\section{Authors' contributions:}

Andrea Morelli planned the study, was responsible for its design and coordination and drafted the manuscript. He had full access to all the data in the study and takes responsibility for the integrity of the data and the accuracy of the data analysis.

Salvatore Mario Romano participated in the study design and data interpretation. Performed the statistical analysis and helped to draft the manuscript.

Mervyn Singer, Vito Marco Ranieri, Giacomo Frati, Mariangela Peruzzi, Ernesto Greco, Luciana Mascia, Fabio Guarracino participated in the data interpretation and helped to draft the manuscript.

Giuseppe Biondi Zoccai contributed to the statistical analysis, the description of the statistical results, the data interpretation and helped to draft the manuscript. 
Alessandra Orecchioni, Annalia D’Egidio, Fernando Piscioneri were responsible for data collection and helped to draft the manuscript.

Critical revision of the manuscript for important intellectual content: Andrea Morelli, Mervyn Singer, Vito Marco Ranieri, Salvatore Mario Romano, Fabio Guarracino, Giacomo Frati

\section{REFERENCES}

[1] Dellinger RP, Levy MM, Rhodes A, et al. (2013) Surviving sepsis campaign: international guidelines for management of severe sepsis and septic shock: 2012. Crit Care Med.; 41:580-637

[2] Guarracino F, Ferro B, Morelli A, Bertini P, Baldassarri R, Pinsky MR. (2014) Ventriculoarterial decoupling in human septic shock. Crit Care;18:R80

[3] Guarracino F, Baldassarri R, Pinsky MR. (2013) Ventriculo-arterial decoupling in acutely altered hemodynamic states. Crit Care; 17:213.

[4] Vieillard-Baron A, Caille V, Charron C, Belliard G, Page B, Jardin F (2008) Actual incidence of global left ventricular hypokinesia in adult septic shock. Crit Care Med; 36:1701-1706.

[5] Ohte N, Cheng CP, Little WC. (2003) Tachycardia exacerbates abnormal left ventricular-arterial coupling in heart failure. Heart Vessels.; 18:136-41.

[6] Prabhu SD. (2007) Altered left ventricular-arterial coupling precedes pump dysfunction in early heart failure. Heart Vessels; 22:170-7. 
[7] Magder SA. (2012) The ups and downs of heart rate. Crit Care Med; 40:239-245.

[8] Azimi G, Vincent JL. (1986) Ultimate survival from septic shock. Resuscitation; $14: 245-253$

[9] Parker MM, Shelhamer JH, Natanson C, Alling DW, Parrillo JE. (1987) Serial cardiovascular variables in survivors and nonsurvivors of human septic shock: heart rate as an early predictor of prognosis. Crit Care Med; 15:923-9.

[10] Morelli A, Ertmer C, Westphal M, et al. (2013) Effect of heart rate control with esmolol on hemodynamic and clinical outcomes in patients with septic shock: a randomized clinical trial. JAMA; 310:1683--91.

[11] Vellinga NA, Boerma EC, Koopmans M, et al. (2015) International study on microcirculatory shock occurrence in acutely ill patients. Crit Care Med; 43:48-56.

[12] Leibovici L, Gafter-Gvili A, Paul M, et al. (2007) Relative tachycardia in patients with sepsis: an independent risk factor for mortality. QJM; 100:629-634.

[13] Dekleva M, Lazic JS, Soldatovic I, et al. (2015) Improvement of ventricular-arterial coupling in elderly patients with heart failure after beta blocker therapy: Results from the CIBIS-ELD Trial. Cardiovasc Drugs Ther; 29:287-94

[14] Razzolini R, Tarantini G, Boffa GM, Orlando S, Iliceto S. (2004) Effects of carvedilol on ventriculo-arterial coupling in patients with heart failure. Ital Heart J; $5: 517-22$

[15] Romano SM, Pistolesi M. (2002) Assessment of cardiac output from systemic arterial pressure in humans. Crit Care Med; 30:1834-41

[16] Scolletta S, Bodson L, Donadello K, Taccone FS, Devigili A, Vincent JL, De Backer D. (2013) Assessment of left ventricular function by pulse wave analysis in critically ill patients. Intensive Care Med; 39:1025-33.

[17] Lewis T. The factors influencing the prominence of the dicrotic wave. (1906) J Physiol; 34:414-29 
[18] Smith D, Craige E. (1986) Mechanism of the dicrotic pulse. Br Heart J. 56:531-4.

[19] Fincke R, Hochman JS, Lowe AM, et al. (2004) Cardiac power is the strongest hemodynamic correlate of mortality in cardiogenic shock: a report from the SHOCK trial registry. J Am Coll Cardiol; 44:340-8.

[20] Sunagawa K, Maughan WL, Burkhoff D, Sagawa K. (1983) Left ventricular interaction with arterial load studied in the isolated canine ventricle. Am J Physiol;245:H733-H788

[21] Freeman GL, Little WC, O’Rourke RA. (1987) Influence of heart rate on the left ventricular performance in conscious dogs. Circ Res; 61:455-464

[22] Rudiger A, Singer M. (2007) Mechanisms of sepsis-induced cardiac dysfunction. Crit Care Med; 35:1599-1608.

[23] Sanfilippo F, Santonocito C, Morelli A, Foex P. (2015) Beta-blocker use in severe sepsis and septic shock: a systematic review. Curr Med Res Opin; 31:1817-25

[24] Pemberton P, Veenith T, Snelson C, Whitehouse T. (2015) Is It Time to Beta Block the Septic Patient? Biomed Res Int:424308

[25] Asfar P, Meziani F, Hamel JF, et al. (2014) High versus low blood-pressure target in patients with septic shock. N Engl J Med; 370:1583-93.

[26] Kimmoun A, Louis H, Kattani NA, et al. (2015) $\beta_{1}$-adrenergic inhibition improves cardiac and vascular function in experimental septic shock. Crit Care Med;43: e332-40

[27] Ogura Y, Jesmin S, Yamaguchi N, et al. (2014) Potential amelioration of upregulated renal HIF-1alpha-endothelin-1 system by landiolol hydrochloride in a rat model of endotoxemia. Life Sci; 118:347-56

[28] Seki Y, Jesmin S, Shimojo N, et al. (2014) Significant reversal of cardiac upregulated endothelin-1 system in a rat model of sepsis by landiolol hydrochloride. Life Sci; 118:357-63

[29] Bergel DH. (1961) The dynamic elastic properties of the arterial wall. J Physiol; $156: 458-469$. 
[30] Giannattasio C, Vincenti A, Failla M, et al. (2003) Effects of heart rate changes on arterial distensibility in humans. Hypertension; 42:253-6.

[31] Repessé X, Charron C, Vieillard-Baron A. (2013) Evaluation of left ventricular systolic function revisited in septic shock. Crit Care;17(4):164 


\section{Figures:}

Fig 1. Study flowchart. CVP, central venous pressure; HR, heart rate; MAP, mean arterial pressure;

NE, norepinephrine; PAOP, pulmonary arterial occlusion pressure; SSC, Surviving Sepsis

\section{Campaign.}

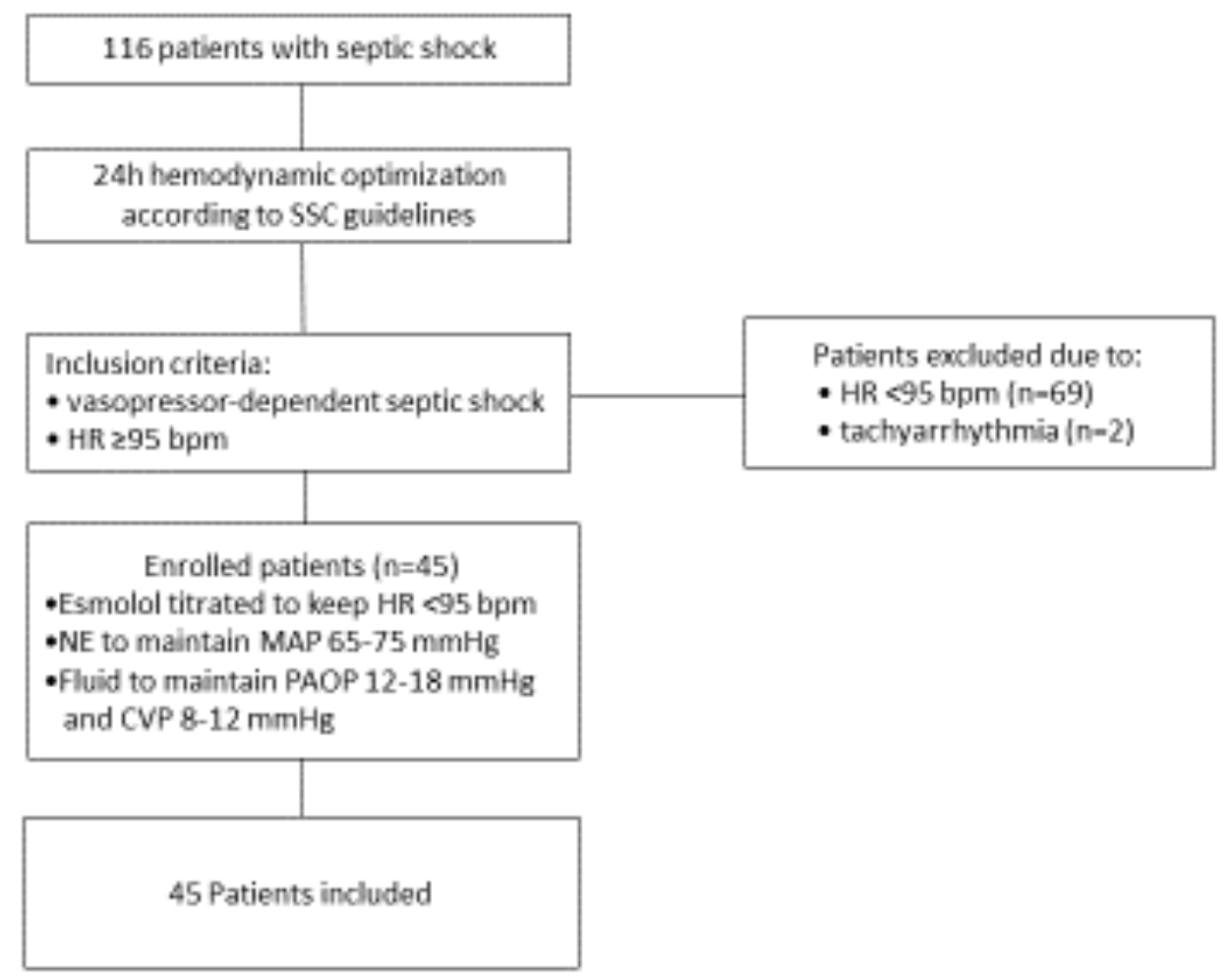


Fig 2. Scatterplot of HR values while performing transesophageal echocardiography at the end of the observational period.

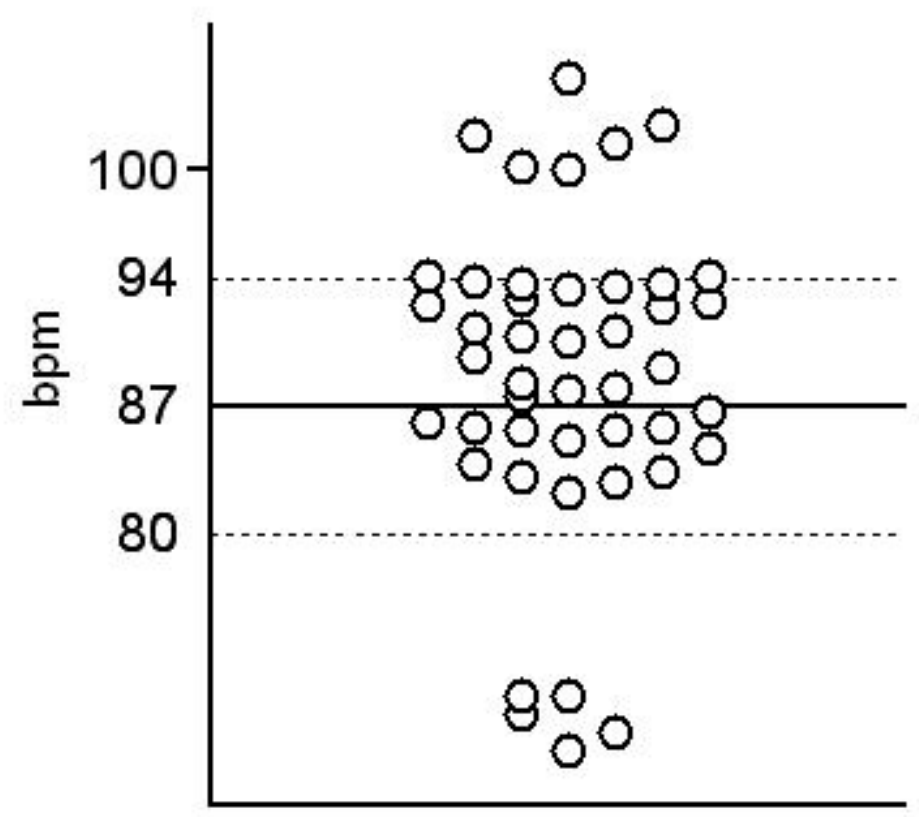


Fig 3. Example of an arterial waveform before (A) and after (B) reducing heart rate. Note the biphasic nature of the flow pattern during tachycardia, and the more physiological waveform after reducing heart rate with esmolol. Changes were achieved through an increase in SV as well as the ability of the cardiovascular system to increase arterial tone in response to an augmented SV.

A

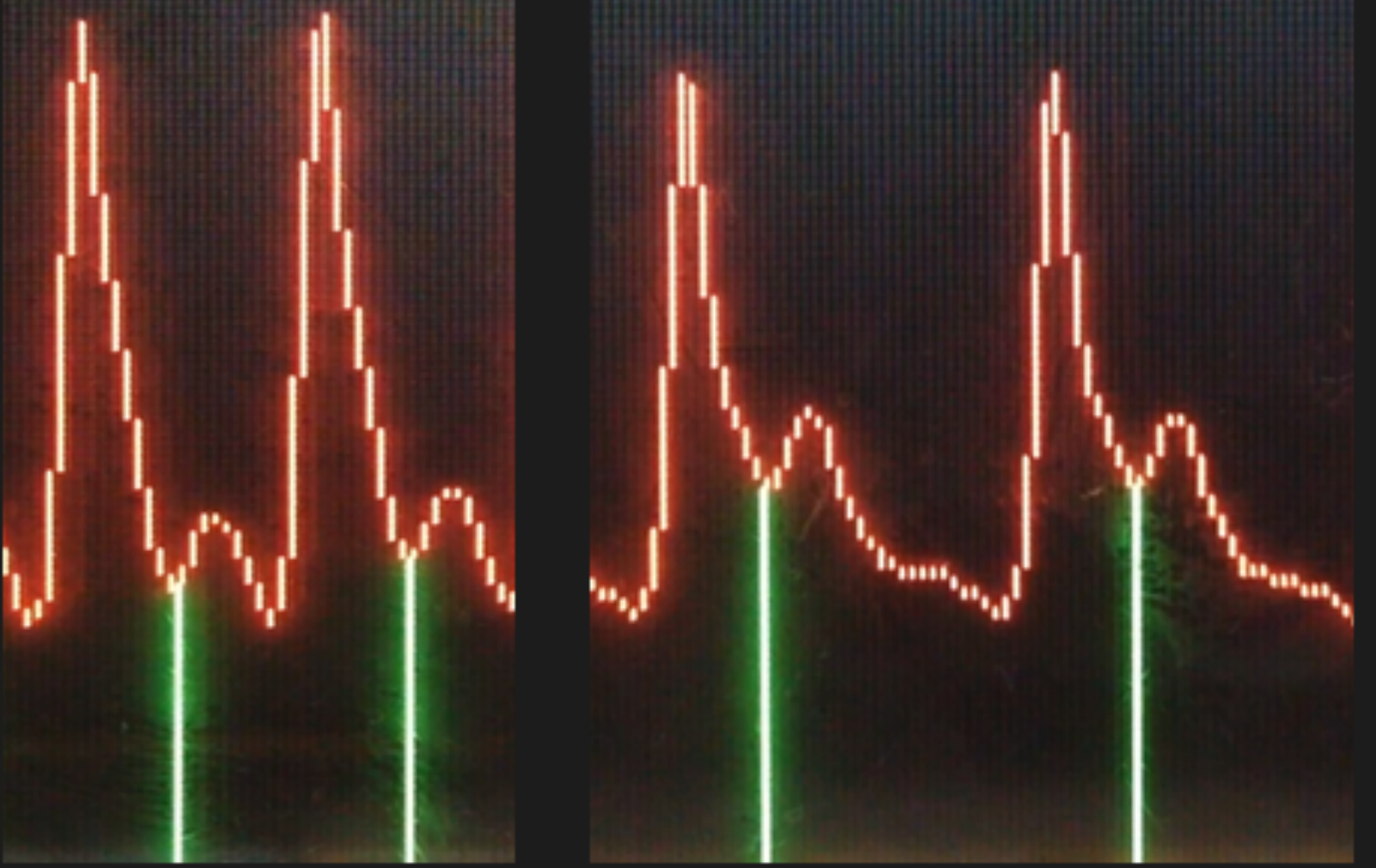


Table 1. Characteristics of the study patients $(n=45)$

\begin{tabular}{ll}
\hline Age, yrs & $61 \pm 18$ \\
Gender, male & $73 \%$ \\
SAPS II & $54 \pm 7$ \\
28 day mortality & $51 \%$ \\
ICU length of stay, days & $18 \pm 17$ \\
\hline
\end{tabular}

Data given as mean and standard deviation. SAPS II, simplified acute physiology score II; ICU, intensive care unit. 
Table 2. Hemodynamic, echocardiographic and arterial waveform data

\begin{tabular}{|c|c|c|c|}
\hline Variable & Baseline & 4 hours & $p$ value \\
\hline $\mathrm{CO}^{\text {th }}\left[\mathrm{L} \cdot \mathrm{min}^{-1}\right]$ & $5.4 \pm 1.3$ & $5.1 \pm 1.4$ & 0.11 \\
\hline $\mathbf{S V}^{\text {th }}[\mathrm{mL}]$ & $48 \pm 14$ & $59 \pm 18$ & $<0.001$ \\
\hline $\operatorname{CO}^{\mathfrak{p}}\left[L \cdot \min ^{-1}\right]$ & $5.1 \pm 1.3$ & $5.0 \pm 1.3$ & 0.77 \\
\hline $\mathbf{S V}^{\mathfrak{p}}[\mathrm{mL}]$ & $47 \pm 12$ & $59 \pm 16$ & $<0.001$ \\
\hline $\mathbf{H R}\left[\mathrm{min}^{-1}\right]$ & $115 \pm 11$ & $88 \pm 9 *$ & $<0.001$ \\
\hline SVR $\left[\right.$ Dyn $\left.\bullet \mathrm{s} / \mathrm{cm}^{5}\right]$ & $1234 \pm 293$ & $1102 \pm 260$ & 0.001 \\
\hline MAP $[\mathrm{mmHg}]$ & $80 \pm 12$ & $75 \pm 10$ & 0.005 \\
\hline MPAP $[\mathrm{mmHg}]$ & $30 \pm 7$ & $28 \pm 6$ & 0.001 \\
\hline PAOP $[\mathrm{mmHg}]$ & $16 \pm 3$ & $16 \pm 4$ & 0.74 \\
\hline CVP [mmHg] & $12 \pm 3$ & $12 \pm 3$ & 0.86 \\
\hline $\mathrm{Ea}^{\mathfrak{p}}\left[\left.\mathrm{mmHg} \cdot\right|^{-1}\right]$ & $2.2 \pm 0.7$ & $1.7 \pm 0.5$ & $<0.001$ \\
\hline $\mathrm{Ea}^{\text {th }}\left[\left.\mathrm{mmHg} \cdot\right|^{-1}\right]$ & $2.0 \pm 0.6$ & $1.55 \pm 0.5$ & $<0.001$ \\
\hline LVEF [\%] & $52 \pm 11$ & $53 \pm 11$ & 0.17 \\
\hline Art $\mathbf{d P} / \mathrm{dt}_{\mathrm{MAX}}\left[\mathrm{mmHg} \cdot \mathrm{ms}^{-1}\right]$ & $1.08 \pm 0.32$ & $0.89 \pm 0.29$ & 0.0009 \\
\hline CCE [units] & $-0.15 \pm 0.5$ & $-0.01 \pm 0.4$ & 0.002 \\
\hline CPwO [W] & $0.53 \pm 0.14$ & $0.63 \pm 0.24$ & 0.007 \\
\hline NE dosage $\left[\mu \mathrm{g} \cdot \mathrm{kg}^{-1} \cdot \mathrm{min}^{-1}\right]$ & $0.7 \pm 0.7$ & $0.58 \pm 0.55$ & 0.01 \\
\hline $\mathbf{P}_{\text {sys }}[\mathrm{mmHg}]$ & $119 \pm 18$ & $110 \pm 18$ & 0.0003 \\
\hline $\mathbf{P}_{\text {dia }}[\mathrm{mmHg}]$ & $61 \pm 12$ & $57 \pm 9$ & 0.0004 \\
\hline $\mathbf{P}_{\text {dic }}[\mathrm{mmHg}]$ & $72 \pm 15$ & $70 \pm 12$ & 0.45 \\
\hline $\mathbf{M A P}-\mathbf{P}_{\mathrm{dic}}[\mathrm{mmHg}]$ & $9.4 \pm 9$ & $4.3 \pm 8$ & $<0.0001$ \\
\hline
\end{tabular}

Data given as mean and standard deviation. $\mathrm{CO}^{\text {th }}$, cardiac output obtained with thermodilution; SV ${ }^{\text {th }}$, stroke volume obtained with thermodilution; $\mathrm{CO}^{\mathrm{p}}$ cardiac output obtained with the pulse contour analysis; $\mathrm{SV}^{\mathrm{p}}$, stroke volume obtained with the pulse contour analysis; HR, heart rate; MAP, mean arterial pressure; MPAP, mean pulmonary arterial pressure; PAOP, pulmonary arterial occlusion pressure; CVP, central venous pressure; $\mathrm{Ea}^{\mathrm{p}}$, arterial elastance obtained with the pulse contour analysis; $\mathrm{Ea}^{\text {th }}$, arterial elastance obtained with thermodilution; LVEF, left ventricular ejection fraction; CCE, cardiac cycle efficiency; CPwO, cardiac power output; $N E$, norepinephrine, $\mathrm{P}_{\text {sys }}$, systolic pressure; $\mathrm{P}_{\mathrm{dia}}$, diastolic pressure; $\mathrm{P}_{\mathrm{dic}}$, dicrotic pressure. ${ }^{*}$ See figure 2 